\title{
The Acoustic Emission Testing Technology on Large Crane Structure Damage
}

\author{
Zhang Yanbing ${ }^{1}$, Yang $\mathbf{L i}^{2}$ \\ ${ }^{1}$ Special Equipment Safety Supervision Inspection Institute of Jiangsu Province, Branch of Nantong, Nantong, China \\ ${ }^{2}$ School of Mechanical Engineering, Southeast University, Nanjing, China
}

Email address:

93829185@qq.com (Zhang Yanbing),1690065668@qq.com (Yang Li)

\section{To cite this article:}

Zhang Yanbing, Yang Li. The Acoustic Emission Testing Technology on Large Crane Structure Damage. Engineering and Applied Sciences. Vol. 5, No. 1, 2020, pp. 9-14. doi: 10.11648/j.eas.20200501.12

Received: January 13, 2020; Accepted: January 27, 2020; Published: February 13, 2020

\begin{abstract}
The Acoustic Emission testing application on Crane steel structure is greatly limited, because of the factors such as the random transmission path of sound wave, the influence of dynamic load noise and the discontinuity of structure. In this paper, the feasibility of Acoustic Emission detection for crane structure damage is discussed from two aspects: mechanism test and field detection. Based on the analysis of the whole test process, when lifting heavy objects and braking, the metal structure is subjected to the instantaneous gravity load of tens of tons, so that a large number of AE signals are collected by various monitoring sensors. It mainly includes active defect damage, mechanical vibration, structural friction and electrical noise, etc. At this time, the effective defect expansion signal is compared with the noise signal. However, in the load maintenance phase, the noise signal disappears or drops to a very low level. At this point, if a sensor still collects a strong active AE signal, it is highly likely that there is a damage source in this area. Compared with the traditional mechanical properties of materials, AE characteristic parameters, such as amplitude, ringing count and energy count, can reflect the microscopic damage changes of materials under load in a more detailed way. By summing up the distribution range of AE parameters corresponding to different damage mechanisms and typical signal characteristics, such as the "double peaks" phenomenon in the material yield stage, it can provide a scientific foundation for the application of AET in the metal structure damage of large lifting machinery.
\end{abstract}

Keywords: Crane, Acoustic Emission (AE), Testing

\section{Introduction}

Acoustic emission technology (AET) [1-3], which uses instruments to detect and analyze elastic waves generated by rapid release of energy during the loading process of materials and thus to make a judgment on the integrity state of materials, has been widely applied in petrochemical, aerospace and other fields. The steel structure of large hoisting machinery is the main matrix of the whole equipment, and its height varies from tens of meters to hundreds of meters [4-6]. For a long time, due to the complex structure of hoisting machinery, the acoustic wave propagation is affected by the discontinuity of the structure and the mechanical noise of the crane itself as a moving device, the application of AE technology in the on-line damage detection of steel structures of large hoisting machinery is limited [7-11]. Therefore, in this paper, the feasibility of AE detection of crane structure damage is discussed and verified from two aspects of material damage AE source mechanism test analysis and field detection.

\section{Experimental Analysis of AE Source Mechanism of Material Damage}

Because the mechanism and characteristics of AE signals are closely related to the microcrack initiation, propagation and plastic deformation in materials, the most commonly used materials of hoisting machinery Q235B are selected for AE monitoring of the whole process of tensile damage. In this way, the mapping correspondence between AE source signal characteristics and material damage is explored, providing basic theoretical data support for the application of AET to hoisting machinery condition monitoring. In the test, two most 
common structural patterns of crane members were selected: welding samples and complete steel plate samples for tensile test.

During the experiment, two channels are arranged at both ends of the sample, and I, II, III and IV are used to mark the four stages of material stretching [12], and the signal characteristics of each stage are analyzed. Comparing the tensile curves of the two samples (see Figure 1), it can be found that in the elastic deformation stage, the load time history curve of the two samples coincides completely because the non welded area mainly bears the occurrence of elastic deformation. However, due to the thermal effect of welding and the change of the internal structure of the material, the elastic deformation of the weld area is small at the low load stage. Moreover, the strength of the weld area is higher than that of the base metal due to the thermal effect of the welding process, so the yield limit of the welded specimen is higher than that of the complete specimen on the curve. On the other hand, the welding process makes the strength of the material increase while the molding decreases, or the maximum deformation energy reserve it can bear decreases, which leads to the shorter strengthening stage of the welding specimen, that is to say, the fracture occurs earlier.
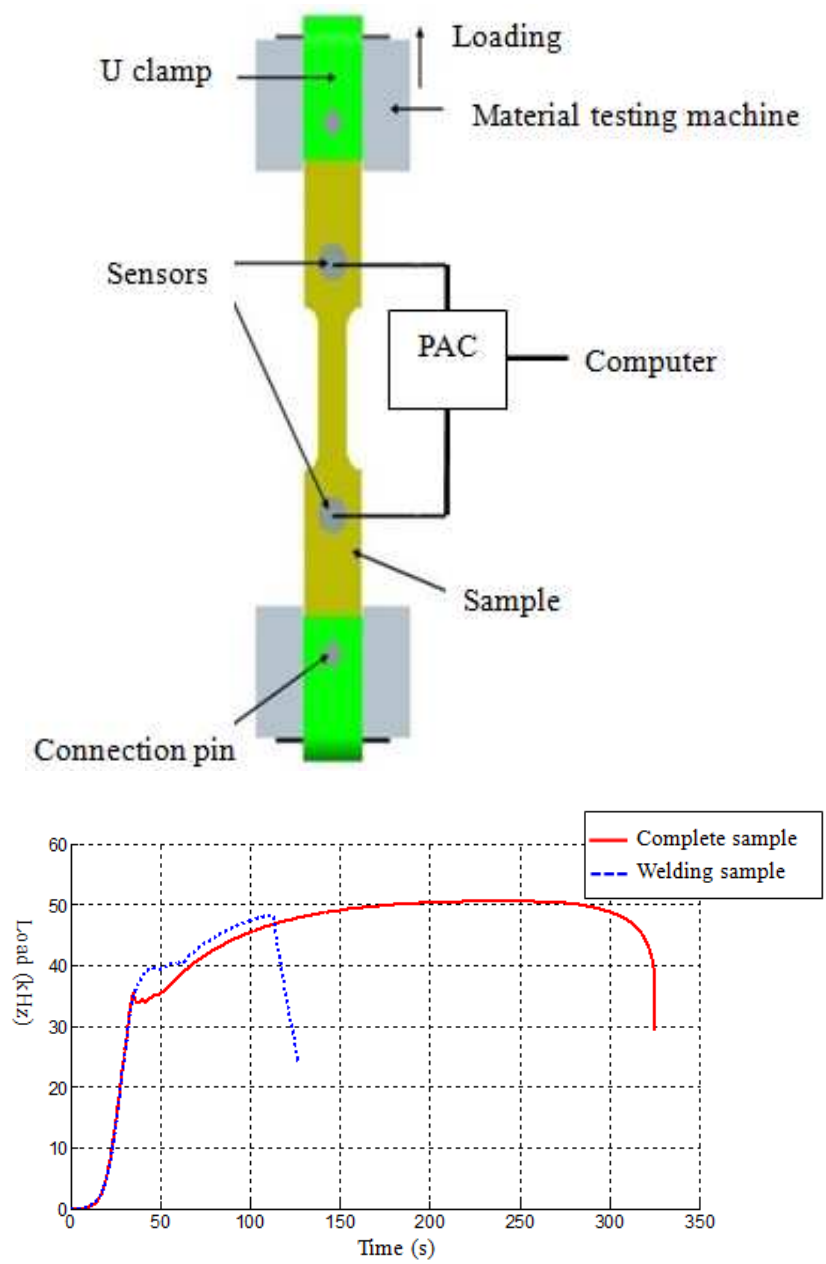

Figure 1. Tensile specimen and its loading process.
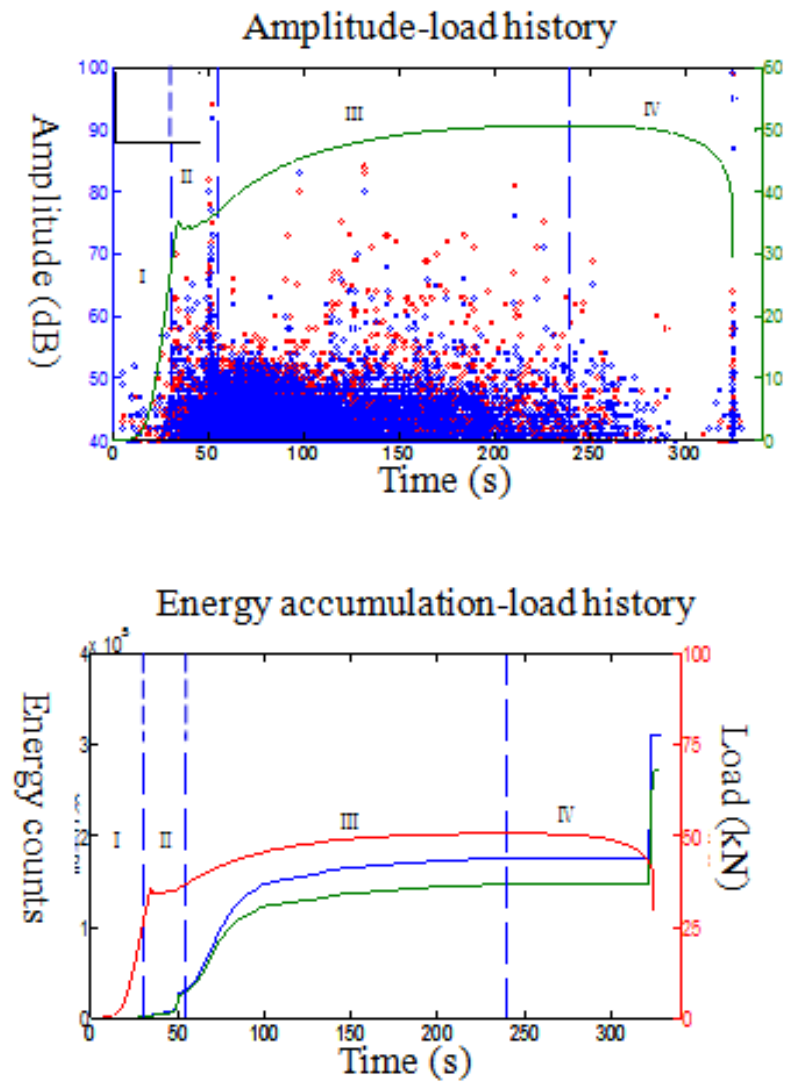

(a) A complete sample
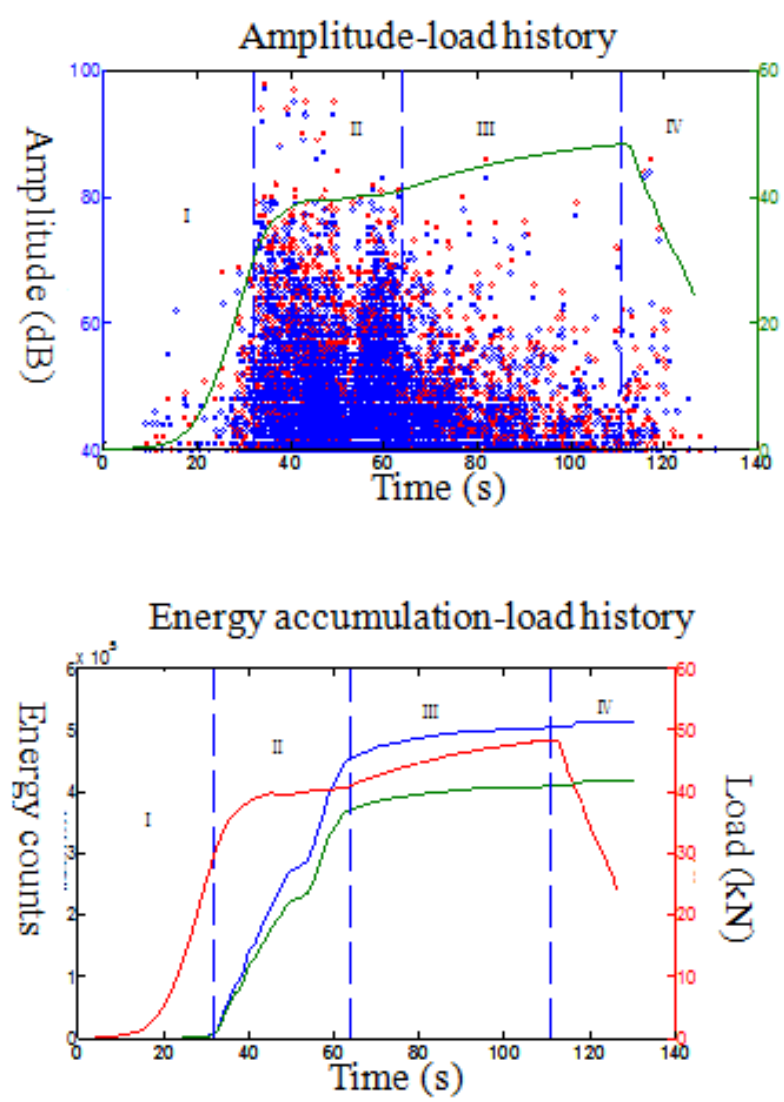

(b) Welding sample

Figure 2. AE amplitude and ringing count-time history of tensile damage. 
(1) Preload elastic deformation stage at this stage, the characteristics of the two samples are basically the same, almost few AE signal occurs, only under the load of more than $60 \%$ yield strength, there are a few signals. However, the energy rate, amplitude, ringing count, RMS, duration and other typical AE characteristic parameters are all at a very low level, which is still far from the typical defect extension signal [13]. This is mainly caused by the structural friction between the fixture, the pin shaft and the sample or the initial loosening of the gap between the internal tissues of the material. In General, this stage more reflects a friction damage process (see Figure 2).

(2) Plastic yielding stage in the early stage of stage II, the amplitude of the signal of the intact sample is relatively low, and the ring count is less. With the material yielding, a strong signal with a maximum amplitude of $94 \mathrm{db}$ appears at $50 \mathrm{~s}$, and the ring count value reaches a peak value of 1768. This is mainly due to the increase of dislocation motion, density, dislocation slip and dislocation avalanche in the yield stage, which leads to the intense energy release of the local stress field in the material. Furthermore, it is characterized by strong activation of AE signal, and when it reaches the upper yield point, the activity and energy value of AE signal reach the peak value.

In the stage I, due to the thermal effect of the welding process, the upper yield point of the welding sample is increased, and the total time of the yield stage is extended. In this stage, the intensity and activity of the AE signal are also higher. In this stage, the two samples show the following characteristics: the number of impact, amplitude and ring count rate of welded samples are higher. This is because the local materials in the welding area achieve a transformation similar to heat treatment strengthening under the action of high temperature, which leads to the need for higher load to reach the yield stage. Secondly, during the process from the upper yield point to the lower yield point (in the 50s), AE parameter values dropped rapidly and there was a short "trough". After passing the lower yield point, the activity of AE signal begins to increase again, but the maximum value is lower than that before the upper yield, which is called "double peak" phenomenon [14].

(3) Reinforcement stage in the third stage, due to the effect of work hardening, the density of dislocations in the material increases, and dislocation packing, entanglement and splitting occur between the metal lattices, which greatly increases the difficulty of dislocation movement, and ultimately increases the load required for the specimen to continue to deform. In the early stage of this process, AE signal has a strong activity, but the amplitude has a very significant decline compared with the yield stage. At the later period of this stage, the AE signal quantity is obviously reduced, and its overall magnitude is gradually reduced. Compared with the two samples, the acoustic emission signal characteristics of the two samples are basically similar at this stage.

(4) Local necking stage at this time, it can be seen from the load curve that the load on the specimen is gradually decreasing, and there is almost no acoustic emission signal in the process, but a very strong transient impact occurs at the moment of specimen fracture.

It can be seen from the above results that the damage of materials has a very high correlation with the characteristics of acoustic emission signals. In order to provide the basis for judging the damage degree of the actual crane AE detection, it is necessary to summarize the AE signal characteristics of different damage modes (see Table 1).

Table 1. AE impact statistics of different amplitude/energy ranges.

\begin{tabular}{|c|c|c|c|c|c|c|c|c|c|}
\hline \multirow{2}{*}{ Stage } & & \multicolumn{4}{|c|}{ Amplitude (dB) } & \multicolumn{4}{|c|}{ Energy } \\
\hline & & $40-50$ & $50-60$ & $60-70$ & $70-100$ & $1-10$ & $10-100$ & $100-1000$ & $>1000$ \\
\hline \multirow{2}{*}{ I } & Complete & 102 & 11 & 0 & 0 & 49 & 60 & 5 & 0 \\
\hline & Welding & 141 & 37 & 11 & 0 & 44 & 121 & 26 & 0 \\
\hline \multirow{2}{*}{ II } & Complete & 1289 & 180 & 33 & 14 & 815 & 633 & 63 & 5 \\
\hline & Welding & 3890 & 1753 & 666 & 240 & 1532 & 3332 & 1581 & 103 \\
\hline \multirow{2}{*}{ III } & Complete & 17349 & 754 & 84 & 22 & 10370 & 7623 & 120 & 6 \\
\hline & Welding & 1387 & 382 & 90 & 32 & 703 & 1003 & 180 & 2 \\
\hline IV & Welding & 109 & 30 & 10 & 8 & 85 & 44 & 20 & 4 \\
\hline
\end{tabular}

\section{AE Detection for Damage of Metal Structure of Gantry Crane}

MQ1260-45 truss gantry crane has been put into use for more than 30 years. In the long-term use, it bears the fatigue load and environmental corrosion, and its steel structure has many cracks, such as the pull rods on both sides of the A-frame, the chord at the root of the boom, and the local narrow area of the turntable girder. In order to verify the influence of these macro defects and potential buried defects on the stability of the whole hoisting machinery structure, the
AET was used to test the safety performance of the steel structure under 1.25 times static load. Because the structure of truss crane is more complex than that of plane equipment (such as pressure vessel), the acoustic signal can't propagate in a straight line in this kind of structure. Therefore, the traditional AE source location methods such as linear location, plane location and so on will not be applicable [15]. Therefore, in the detection process, the AE point by point monitoring method is used to realize the detection of specific areas, such as places with large working stress and severe stress concentration area on the crane structure (see Figure 3), so as to realize the detection of the damage degree. 


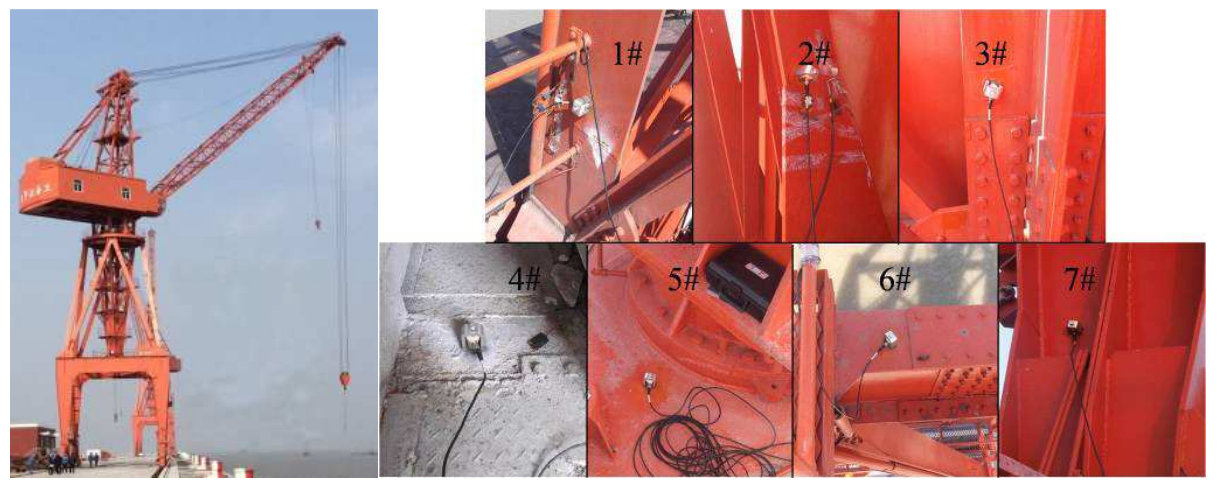

Figure 3. Layout of field detection sensor.

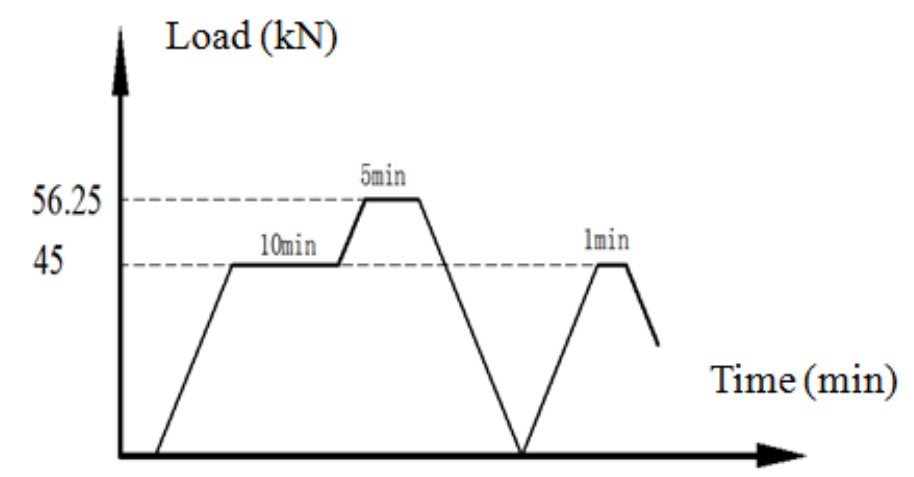

Figure 4. AE test loading program.
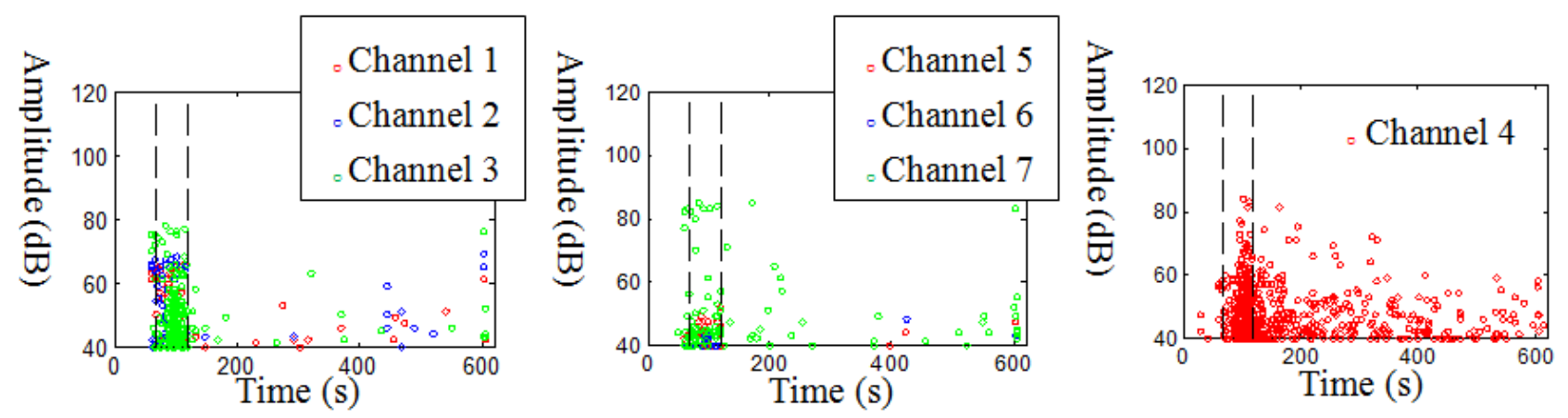

Figure 5. Amplitude history diagram of each channel in the process of 0-45t load lifting.

During the static load test of the crane, the whole process of on-line detection is divided into two loading cycles (see Figure 4). In the detection results, the dotted line is used as the time separation, and the lifting and braking are started according to the time sequence.

(1) Before lifting heavy objects, effective AE signals were not collected in all channels, indicating that various environmental noises affecting the detection results were well suppressed.

(2) At the beginning of hoisting, a large number of $\mathrm{AE}$ events appeared in each channel at the instantaneous concentration. With the increase of lifting height, the characteristic parameters of AE signal also increased rapidly, and braking was conducted after reaching the expected lifting height. During this process, AE signals showed very high activity. Among them, the peak amplitudes of acoustic emission signals in the three regions of the main chord of the rotating column (3\#), the bottom leg of the door frame (7\#) and the girder of the rotating platform (4\#) in the machine room are respectively $79 \mathrm{~dB}, 86 \mathrm{~dB}$ and $85 \mathrm{~dB}$. However, the AE signal in other regions monitored at the same time was relatively weak, indicating that the No. 3, 4 and 7 sites were subjected to greater load impact under the action of lifting weights.

(3) After the first lift was completed, braking was carried out and the load was maintained for $10 \mathrm{~min}$. At this time, the acoustic emission signal in the area corresponding to the No. 4 sensor did not completely disappear, and some AE signals with high strength and activity still appeared, with the amplitude as high as $60-80 \mathrm{~dB}$, while other channels rapidly decreased to below $60 \mathrm{~dB}$ (see Figure 5). 


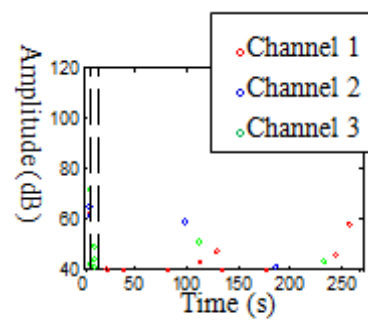

(a) Channel $1 \sim 3$

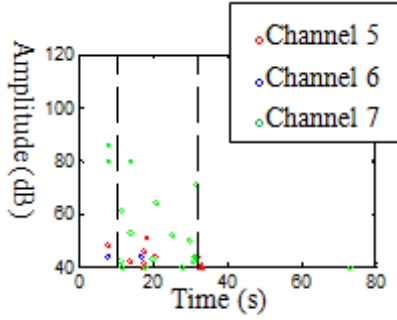

(b) Channel $5 \sim 7$

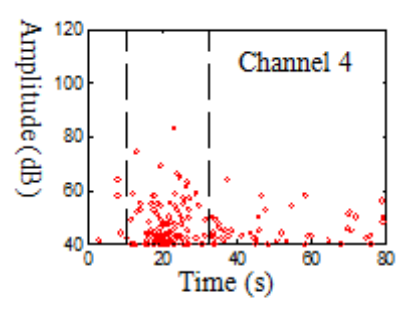

(c) Channel 4

Figure 6. Amplitude history of each channel during 45-56.25t load lifting.

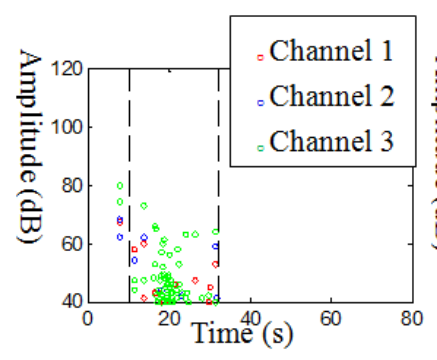

(a) Channel $1 \sim 3$

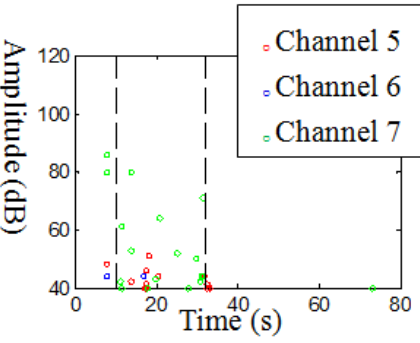

(b) Channel $5 \sim 7$

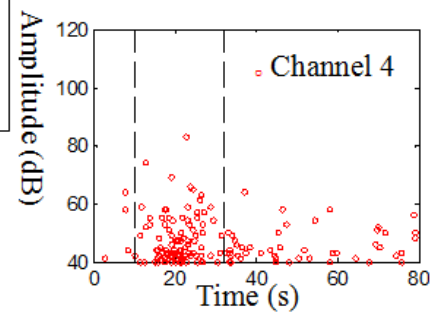

(c) Channel 4

Figure 7. Amplitude history of each channel during the second 0-45t load lifting.

(4) In the second lifting process of 45-56.26t, the impact of the load decreased significantly because the lifting increment of the load was 0.25 times the rated load. Moreover, since the stress between the crane structures has been fully released during the first heavy lifting process, the $\mathrm{AE}$ signals collected at this stage significantly decreased compared with the first lifting. During the first stage of lifting, the crane was unloaded and reloaded. The third lift of this process was due to the Kaiser effect of metal material. The AE signals generated in this stage are also greatly reduced compared with the loading process in the first stage. In the last two load retaining processes, AE signal characteristics of each channel are similar to those of the first stage, only channel 4 still maintains strong activity and strength (see Figure 6).

Based on the analysis of the whole test process, when lifting heavy objects and braking, the metal structure is subjected to the instantaneous gravity load of tens of tons, so that a large number of $\mathrm{AE}$ signals are collected by various monitoring sensors. It mainly includes active defect damage, mechanical vibration, structural friction and electrical noise, etc. At this time, the effective defect expansion signal is compared with the noise signal. However, in the load maintenance phase, the noise signal disappears or drops to a very low level. At this point, if a sensor still collects a strong active AE signal, it is highly likely that there is a damage source in this area. Therefore, combining the results of the three load lifting and holding stages, it can be seen that channel 4 (at the girder of the rotating platform) has a large number of active AE signals due to the presence of several loading and holding loading stages. In addition, it is speculated that there may be some damage inside the material in this area, so it is recommended to use other conventional detection methods to reinspect this area (see Figure 7).

\section{Conclusion}

(1) Compared with the traditional mechanical properties of materials, AE characteristic parameters, such as amplitude, ringing count and energy count, can reflect the microscopic damage changes of materials under load in a more detailed way. By summing up the distribution range of $\mathrm{AE}$ parameters corresponding to different damage mechanisms and typical signal characteristics, such as the "double peaks" phenomenon in the material yield stage, it can provide a scientific foundation for the application of AET in the metal structure damage of large lifting machinery.
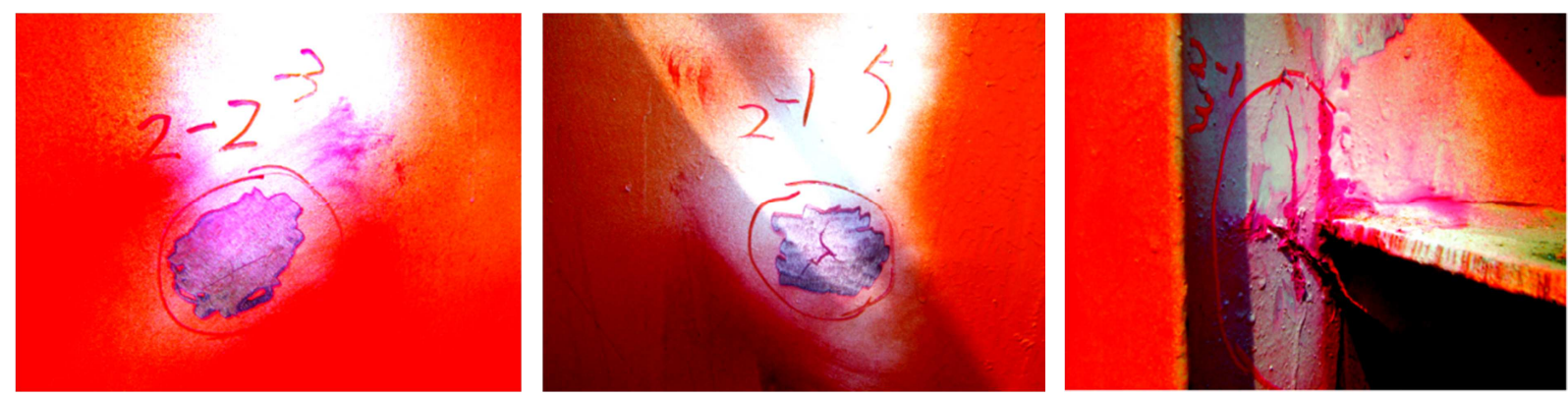

Figure 8. Cracking defects of the main beam of the rotating platform. 
(2) Grinding and penetrant flaw detection were carried out on the rotary girder found in the acoustic emission detection process of the actual gantry crane. Multiple surface cracks and obvious cracks were found on the outer surfaces of the two box girder under the rotary platform (sensors were placed above the girder, i.e. in the top machine room) (see Figure 8). The reason is that this area is the web position of the main beam of the rotary table. Because it bears the cyclic load during the frequent lifting process of the crane for a long time, and this area is located in the stress concentration area of the cantilever beam root, the fatigue damage finally produces cracks.

\section{Acknowledgements}

This research is supported by the National Natural Science Foundation of China (Grant No. 51175078), funded by jiangsu bureau of quality and technical supervision (KJ (Y) 2012014) and special funds for basic scientific research operating expenses of central universities and colleges (CXLX12-0079). The author would appreciate the anonymous reviewers and the editor for their valuable comments.

\section{References}

[1] Dalton PR, Cawley P. and Lowe M. J. Propagation of acoustic emission signals in metallic fuselage structure [J]. IEE Proc-Sci. Meas Technol.. 2001, 148 (4): 167-177.

[2] Mba D, Hall L D. The transmission of acoustic emission across large-scale turbine rotors [J]. NDT \& E International, 2002, 35 (8): 529-539.

[3] Naber R-R, Bahai H. Analytical and experimental validations of a numerical band-limited Green's function approach for modeling acoustic emission waves [J]. Advances in Engineering Softwave, 2007, 38 (112): 876-885.

[4] Herrera A Y, Calero J M, Porras-Montenegro N. Pressure, temperature, and thickness dependence of transmittance in a 1D superconductor-semiconductor photonic crystal [J]. Journal of Applied Physics, 2018, 123 (3): 033101.
[5] Alvey C, Pfeifer C, Irianto J, et al. Mechanosensing of Solid Tumors by Cancer-Attacking Macrophages [J]. Biophysical Journal, 2018, 114 (3): 654a.

[6] Serra P, Oosterloo T, Morganti R, et al. The ATLAS (3D) project-XIII. Mass and morphology of $\mathrm{HI}$ in early-type galaxies as a function of environment [J]. Monthly Notices of the Royal Astronomical Society, 2011, 422 (3).

[7] Yao Y, Fine M E, Keer L M. An energy approach to predict fatigue crack propagation in metals and alloys [J]. International Journal of Fracture, 2016, 146 (3): 149-158.

[8] Ferreira S E, Castro J T P D, Meggiolaro M A. Using the strip-yield mechanics to model fatigue crack growth by damage accumulation ahead of the crack tip [J]. International Journal of Fatigue, 2017, 103: 557-575.

[9] Brunner, Andreas J. Identification of damage mechanisms in fiber-reinforced polymer-matrix composites with Acoustic Emission and the challenge of assessing structural integrity and service-life [J]. Construction and Building Materials, 2018, 173: 629-637.

[10] Castelluccio G M, Mcdowell D L. Microstructure-sensitive small fatigue crack growth assessment: Effect of strain ratio, multiaxial strain state, and geometric discontinuities [J]. International Journal of Fatigue, 2016, 82: 521-529.

[11] Jiang Y, Xu F, Xu B. Acoustic Emission tomography based on simultaneous algebraic reconstruction technique to visualize the damage source location in Q235B steel plate [J]. Mechanical Systems and Signal Processing, 2015, 64-65: 452-464.

[12] Zheng XL. Mechanical behavior of engineering materials [M]. Xi'an: northwestern polytechnical university press, 2004.

[13] Li MY, Shang ZD, et al. Acoustic emission detection and signal processing [M]. Beijing: Science press, 2010.

[14] Zhang YH, Zhang WB, Zhang YB, et al. Acoustic emission characteristics of Q235B steel plate during tensile damage test [J]. Journal of vibration and shock, 2015, 34 (15): 156-161.

[15] $\mathrm{Wu} \mathrm{ZW,} \mathrm{Shen} \mathrm{GT,} \mathrm{et} \mathrm{al.} \mathrm{The} \mathrm{status} \mathrm{of} \mathrm{acoustic} \mathrm{emission}$ technology in crane nondestructive testing [J]. Hoisting and conveying machinery, 2007 (10): 4. 